\title{
The algorithm development based on the immune search for solving unclear problems to detect the optical flow with minimal cost
}

\author{
Olga Purchina ${ }^{1}$, Anna Poluyan ${ }^{1}$, and Dmitry Fugarov ${ }^{1, *}$ \\ ${ }^{1}$ Don State Technical University, Rostov-on-Don, Russia
}

\begin{abstract}
The main aim of the research is the development of effective methods and algorithms based on the hybrid principles functioning of the immune system and evolutionary search to determine a global optimal solution to optimisation problems. Artificial immune algorithms are characterised as diverse ones, extremely reliable and implicitly parallel. The integration of modified evolutionary algorithms and immune algorithms is proposed to be used for the solution of above problem. There is no exact method for the efficient solving unclear optimisation problems within the polynomial time. However, by determining close to optimal solutions within the reasonable time, the hybrid immune algorithm (HIA) is capable to offer multiple solutions, which provide compromise between several goals. Quite few researches have been focused on the optimisation of more than one goal and even fewer used to have distinctly considered diversity of solutions that plays fundamental role in good performance of any evolutionary calculation method.
\end{abstract}

\section{Introduction}

The existing application of artificial immune algorithms (AIA) for optimisation problems is usually studied as a competitive adaptive mechanism for the artificial neural networks, in which the adaptation mechanism becomes active in case of approaching threat followed with the further output accumulation. Artificial immune algorithms are also used to solve the problems connected with the anomalies identification and detection. This research studies the symbiosis of the artificial immune algorithms, based on the bionic search [1].

There are certain advantages of artificial immune algorithms to bionic search systems as the follows: wide variety, high reliability, implicit parallelism. At the same time there are some disadvantages of AIA: training complexity, difficulties by choosing offspring for cloning and mutation, multiplicity of initial parameters at the start of functioning algorithm, determination of parent chromosomes to form offsprings, etc.

\footnotetext{
*Corresponding author: ddf_1@mail.ru
} 


\section{Research Method}

There should be considered the problem how to find the optimal flow of the minimum cost of the transport network, which is an essential direction in the research of artificial intelligence systems [2].

The parameters of the constructing process of the abilities of access to transport streams are distinguished and the model for the representation of input information is formed on its basis, as well as mathematical model with ill-caused conditions and quality indicators of the obtained solution. The parameters mentioned above are just preconditions and an appropriate algorithm for solving problems of the given type is built or selected on its basis [3].

The minimum cost flow problem is formed in the following way:

$$
\begin{gathered}
F \cong \sum_{(i j) \in U} \sum_{(i j) \in U} \sum_{(i j) \in U} c_{i j} x_{i j} \rightarrow \text { min }, \\
\sum_{(i j) \in U} x_{i j}-\sum_{(i, j) \in U} x_{j k}=\left\{\begin{array}{c}
-r, j=\alpha \\
0, j \neq \alpha, \beta, \\
r, j=\beta
\end{array},\right. \\
0 \leq p_{i j} \leq x_{i j} \leq 1_{i j},
\end{gathered}
$$

where $\mathrm{l}_{\mathrm{ij}}$ is the upper limit of the flow; $\mathrm{p}_{\mathrm{ij}}$ is the lower limit of the flow; $\mathrm{c}_{\mathrm{ij}}$ is the unclear cost flow unit delivery; $\cong$ is the unclear equality operation; $r$ is the flow no exceeding set data.

The main idea of HIA is to obtain multiplicity of quasi-optimal solutions (population) with the help of fast probabilistic algorithm and then to create evolution based on the bionic algorithms (BA) and/or artificial immune algorithms (AIA) [4].

In the process of initial population forming, sets of alternative solutions are obtained by cloning solutions, based on heuristic algorithms, and certain number of antibodies is introduced, too. The formation process is going continuously until the average suitability of the population is close to the initial one, that means stability of the network [5]. New antibodies are introduced in case of unstable network. Antibodies are evolving through cloning selection theory that consists of solutions search in the space. The given approach allows one to maintain the population diversity [6].

The optimal population quantity can be calculated with the formula:

$$
\mathrm{N}_{\mathrm{t}+1}=\mathrm{N}_{\mathrm{t}} \mathrm{e}^{\mathrm{r}\left(1-\frac{\mathrm{N}_{\mathrm{t}}}{\mathrm{K}}\right)}
$$

where $\mathrm{N}_{\mathrm{t}}$ is the population quantity; $\mathrm{r}=$ const is the population growth rate, with values of 0 $<\mathrm{r}<2$ from the best range for the population existence within the system; $\mathrm{K}$ is the definite population quantity at which the actual reproduction rate is reduced in the result of competition so that the population as a whole can only restore its quantity in each generation [7].

The work algorithm of HIA can be represented in the following way:

1. Population formation and estimation of the optimal population quantity.

2. Entering the cycle, when the condition is fulfilled:

(2.1) determination of the target function values for each chromosome and the average value of the target function of the entire population and also the danger signals for the antibodies;

(2.2) sampling ( $\mathrm{n}, \%$ ) or quality $\mathrm{n}$ of the best individuals to clone. The quality of clones is connected with affinity;

(2.3) the implementation of modified genetic operators is carried out in the process of adaptation and regulation; 
(2.4) the mutation operator based on the golden section is done, at the same time it can be adjusted gradually;

(2.5) random generation of certain quantity of antibodies, which are added to the population;

(2.6) determination of the target function values to obtain offspring chromosomes, selection of the percentage of best specified chromosomes and addition them to the population with determination of the danger signals;

(2.6.1) the danger zone is determined, dangerous signals are calculated for each antibody and then antibodies concentration is adjusted through its danger signals;

(2.6.2) cloning is performed, a crossing-over operator generates clones' groups, a certain quantity duplicates from random antibodies, and then a mutation operator for each clone is performed keeping the parent antibody;

(2.6.3) the antibody with high availability is selected, which then placed in the danger zone of the parent antibody and antibodies with higher availability than the parent antibody are found from outside the danger zone of the parent antibody;

(2.6.4) the randomly generated antibodies are added, adjusting the population quantity, recalculating the danger signals of all antibodies with following removing antibodies with zero concentration;

(2.7) chromosomes that do not match the specified conditions are removed from the population.

3. Checking the condition of the stop of calculation procedure; if the condition is not fulfilled, then pass to point (2.1), otherwise output of the obtained optimal (quasi-optimal) solution and pass to item 4 .

4. Analysis of the obtained results.

5. End of the algorithm.

\section{Results and Discussion}

The alternative adaptation allows one to intellectualise the control of work algorithm for solving the optimisation problem (here genetic algorithm) [8]. The given approach makes it possible to set specific control alternatives, depending on the algorithm of solving the optimisation problem [9]. The adaptation change realised through decreasing/increasing the number of conditions corresponding to each alternative, gives the control over the inertia of the immune response.

In above algorithm, an antibody is subjected to selection, if it satisfies the condition:

$$
\mathrm{f}\left(\mathrm{Ab}_{\mathrm{i}}\right) \geq 98 \% \times \mathrm{CSel},
$$

where

$$
\text { CSel }=\frac{\sum_{\mathrm{i}-1}^{\mathrm{n}} \mathrm{f}\left(\mathrm{Ab}_{\mathrm{i}}\right)}{\mathrm{n}} .
$$

The convergence between antibodies and antigens is strongly coupled with those ones which are suitable solutions to the problem [10]. This is expressed in terms of a convergence $\left(\mathrm{Ab}_{\mathrm{i}}\right)$ and a normalised function representation $\mathrm{f}\left(\mathrm{Ab}_{\mathrm{i}}\right)$.

The convergence $A b_{i}$ is calculated as [11]

$$
\operatorname{affinity}\left(A b_{i}\right)=\left\{\begin{array}{cl}
\frac{f\left(A b_{i}\right)-f_{\min }}{f_{\max }-f_{\min }}, & P=\max f(x), \\
1-\frac{f\left(A b_{i}\right)-f_{\min }}{f_{\max }-f_{\min }}, & p=\min f(x),
\end{array}\right.
$$


where $\mathrm{f}\left(\mathrm{Ab} \mathrm{b}_{\mathrm{i}}\right)$ is the antibody function, $\mathrm{f}_{\min }$ is the minimal meaning of population, $\mathrm{f}_{\max }$ is the maximal meaning of population.

After AIA obtainment, the danger signals, based on changes of the adaptive environment, may lead to an immune response [12]. Actually the danger signals create a danger zone around themselves and immune cells inside this zone will be activated to participate in the immune response [13].

Since antigens are invisible for the optimisation problem, it might be assumed that each antibody is a peak; at the same time, the area around the peak is a danger zone.

The danger zone is expressed as

$$
\mathrm{D}\left(A \mathrm{~b}_{\mathrm{i}}\right)=\left\{A \mathrm{~b}_{\mathrm{i}} \mid \operatorname{dis}\left(A \mathrm{~b}_{\mathrm{i}}, \mathrm{Ab} \mathrm{b}_{\mathrm{j}}\right)<\mathrm{r}_{\text {danger }}\right\},
$$

where $r_{\text {danger }}$ is the danger zone radius, its meaning corresponds to the peak size.

A danger signal can be interpreted as the received information on the values of target function (a number of "unfavourable" decisions falling into the danger zone). As a result, those solutions are stimulated which fall into the danger zone. Taking into account this idea, a danger signal can be defined as an identifier in the user's interests. It brings to conclusion that it is possible to imagine various scenarios, in which a danger signal could be useful.

In such a way through the selection theory combination for cloning and danger signals, it is possible to model the biological immune mechanism more suitably and precisely. All individuals in the population are combined into a network, which improves similarity of the population in the process of constant evolution [14].

The antibody concentration is dynamic and dependent on the antibody danger signal and the convergence between antibody and antigen. These two factors are the general reasons for the dynamic changes of antibody concentration.

$\alpha \in[0,1]$ is the initial concentration set for the prior population. The antibody concentration decreases down to 0 , when a danger signal comes, otherwise the concentration increases up to 1 . The antibodies concentration in the population in the danger zone reflects the condition of the whole population for the optimisation task and provides the population diversity. The following shutdown criteria for the operation of the HIA are the main termination conditions:

(i) the specified quantity of algorithm generations has been reached;

(ii) the current solution time is longer than the specified one;

(iii) the specific exactness of the solution $\varepsilon$ is achieved, that is within several iterations the difference in the values of the objective function is equal to or less than the specific value of $\varepsilon$.

\section{Conclusions}

The given research represents a hybrid immune algorithm to solve the problem of finding the minimum cost of the optimal flow of transport network, which is a symbiosis of the modified genetic and immune algorithms and operators allowing us to control the adaptation parameters by executing the algorithm, taking into account the influence of possible changes in the boundary conditions.

The approach to the formation and regulation of the population size becomes distinctive feature, which has shown its effectiveness for solving this type of problem. As a result, a formula for the optimal calculation of the number of the population was proposed, the algorithm maintains a good diversity of the population, it has fewer errors, allows one to reduce the search area for affordable solutions and to obtain optimal results within reasonable time. In the given approach each generation of a population consists of two parts. It contains antibodies with memory of the previous generation while the other 
contains randomly added new antibodies. Antibodies with high convergence after mutation operation are found mainly in the vicinity of the parental antibody. After the cloning operation, the convergence of the population is higher than in the previous generation. Antibodies with greater convergence can change the adaptive environment and then generate danger signals. The process of the generation increases, if antibodies with low convergence cannot leave the danger zone, their concentration decreases down to 0 after that they are going to be removed.

Highly convergent antibodies will be preserved in memory due to unchanged conditions. In this mechanism, antibodies in the memory of the population generally have high convergence. This will be ensured by adding new random antibodies to each generation not from the danger zone memorised by antibodies [15]. Thus they will develop a new search location and ultimately the algorithm will find all the peaks in the evolution process.

\section{References}

1. Y.O. Chernyshev, et al., Journal of Theoretical and Applied Information Technology 81(3), 466 (2015)

2. A.Yu. Poluyan, et al., J. Phys. Conf. Ser. 1333, 032056 (2019) https://iopscience.iop.org/article/10.1088/1742-6596/1333/3/032056

3. D.D. Fugarov, Y.Y. Gerasimenko, et al. J. Phys. Conf. Ser. 1118, 012055 (2018) https://iopscience.iop.org/article/10.1088/1742-6596/1118/1/012055

4. A.Yu. Poluyan, et al., J. Phys. Conf. Ser. 1333, 032057 (2019) https://iopscience.iop.org/article/10.1088/1742-6596/1333/3/032057

5. D.D. Fugarov, et al., J. Phys. Conf. Ser. 1333, 062020 (2019) https://iopscience.iop.org/article/10.1088/1742-6596/1333/6/062020

6. A.Y. Poluyan et al., J. Phys. Conf. Ser. 1015, 022013 (2018) https://iopscience.iop.org/article/10.1088/1742-6596/1015/2/022013

7. K.Yu. Solomentsev et al., J. Phys. Conf. Ser. 1015, 032179 (2018) https://iopscience.iop.org/article/10.1088/1742-6596/1015/3/032179

8. Y. Gerasimenko et al., Advances in Intelligent Systems and Computing 1259 AISC, $471(2021)$

9. Y.O. Chernyshev, et al., Journal of Theoretical and Applied Information Technology, 80(1), 13 (2015)

10. D.A. Onyshko, et al., E3S Web of Conferences 164, 03030 (2020) https://www.e3sconferences.org/articles/e3sconf/abs/2020/24/e3sconf_tpacee2020_03030/e3sconf_tpa cee2020_03030.html

11. M. Ganzhur, et al. E3S Web of Conferences 175, 05038 (2020) https://www.scopus.com/record/display.uri?eid=2-s2.0-

85087864586 \& origin $=$ resultslist

12. A. Gazizov, et al., E3S Web of Conferences 210, 11001 (2020) https://www.scopus.com/record/display.uri?eid=2-s2.085098519557 \&origin=resultslist)

13. A.I. Sukhinov et al., Mathematical Models and Computer Simulations 12(2), 232-245 (2020)

https://www.scopus.com/record/display.uri?eid=2-s2.085083258953 \&origin $=$ resultslist

14. O. Agibalov, E3S Web of Conferences 224, 01008 (2020) 
https://www.scopus.com/record/display.uri?eid=2-s2.0-

85098855817 \&origin $=$ resultslist

15. N.N. Ventsov et al., International Conference on Industrial Engineering, Applications and Manufacturing, ICIEAM 2017, $8076476 \quad$ (2017)

https://www.scopus.com/record/display.uri?eid=2-s2.0-

85039958580 \&origin $=$ resultslist 